\title{
The One-dimensional Coulomb-force Problem in Classical Radiation Reaction Theories
}

\author{
W. Heudorfer and M. Sorg \\ Institut für Theoretische Physik der Universität Stuttgart
}

(Z. Naturforsch. 32 a, 685-691 [1977]; received April 28, 1977)

\begin{abstract}
Numerical solutions of the recently proposed equations of motion for the classically radiating electron are obtained for the case where the particle moves in a one-dimensional Coulomb potential (both attractive and repulsive). The solutions are discussed and found to be meaningful also in that case, where the well-known Lorentz-Dirac equation fails (attractive Coulomb force). Discrete, stationary states are found in a non-singular version of the Coulomb potential. During the transition between those stationary states the particle looses energy by emission of radiation, which results in a smaller amplitude of the stationary oscillations.
\end{abstract}

\section{Introduction and Survey of Results}

The widespread dissatisfaction ${ }^{1-10}$ with the Lorentz-Dirac-Rohrlich theory ${ }^{11,12}$ of the classically radiating electron has motivated us to propose the following equations of motion ${ }^{13-15}$ in place of the Lorentz-Dirac equation:

$$
\begin{gathered}
m_{\exp } c^{2}\left\{\hat{\dot{u}}^{\lambda}-(u \hat{\dot{u}}) u^{\lambda}\right\}=K^{\lambda}, \\
m_{\text {mech }} c^{2} \dot{u}^{\lambda}+m_{\mathrm{el}} c^{2}\left\{\hat{\dot{u}}^{\lambda}-(u \hat{\dot{u}}) u^{\lambda}\right\}=K^{\lambda}, \\
m_{\text {mech }} c^{2} \dot{u}^{\lambda}+m_{\mathrm{el}} c^{2}\left\{\frac{\Delta u^{\lambda}}{2 \Delta s}-\left(u \frac{\Delta u}{2 \Delta s}\right) u^{\lambda}\right\}=K^{\lambda},
\end{gathered}
$$

where obvious abbreviations have been used $\left(u^{\lambda} u_{\lambda}\right.$ $=+1)$

$$
\begin{gathered}
\hat{\dot{u}}^{\lambda}:=\mathrm{d} u^{\lambda}{ }_{(s-\Delta s)} / \mathrm{d} s, \\
\Delta u^{\lambda}:=u_{(s)}^{\lambda}-u^{\lambda}{ }_{(s-2 \Delta s)} .
\end{gathered}
$$

The constant non-locality parameter $(\Delta s)$ is connected with the electromagnetic mass $\left(m_{\mathrm{el}}\right)$ by

$$
m_{\mathrm{el}} c^{2} \Delta s=\frac{2}{3} Z^{2} .
$$

The most striking advantages of these new equations with respect to the well-known Lorentz-Dirac equation ${ }^{11}$

$$
m_{\exp } c^{2} \dot{u}^{\lambda}=K^{\lambda}+\frac{2}{3} Z^{2}\left\{\ddot{u}^{\lambda}+(\dot{u} \dot{u}) u^{\lambda}\right\}
$$

have been fully presented in the quoted papers, and a more general framework for extended radiating particles has also been found in the meantime ${ }^{16}$ such that Eqs. (A), (B), (C) appear as being due to differently structured particles characterized by certain "structure functions".

Reprint requests to Dr. M. Sorg, Institut für Theoretische Physik der Universität, Pfaffenwaldring 57, D-7000 Stuttgart 80 .
Since the improvements elaborated so far are based on rather general considerations (for instance: strict validity of causality, non-existence of runaway solutions), we want now to apply the new equations to some concrete, physically relevant problems. In the present paper, we study the solutions of Eqs. (A), (B), (C) for the motion in a Coulomb potential $\left(\Phi_{(r)}=Z^{*} / r\right)$, both attractive and repulsive; and we shall restrict ourselves to one-dimensional (rectilinear) motion. Two-dimensional problems are treated in a forthcoming paper.

The main results of the present paper are the following:

(i) Whereas the Lorentz-Dirac equation $(I, 4)$ is believed to admit no physically meaningful solution at all for the attractive case ${ }^{17}$, we find meaningful and well-behaved solutions for Equations (B), (C).

(ii) In the repulsive case, the solutions of (A) and (B) become unreasonable for high impact velocities in the sense that the final velocity is predicted to be greater in absolute value than the impact velocity. Whereas this deficiency can by removed for Eq. (A) by a slight modification of the force expression $K^{\lambda}$, it remains unavoidable for Equation (B). However, the unwanted effect emerges not until one passes to ultra-relativistic impact velocities; for non-relativistic and weakly relativistic velocities there arises no trouble with Equation (B). On the other hand, Eq. (C) predicts absolutely meaningful particle trajectories at every velocity scale.

(iii) We have altered the singular Coulomb potential $\left(\Phi_{(r)}=Z^{*} / r\right)$ into a non-singular version $\left[\Phi_{(r)}=Z^{*}\left(r_{0}^{2}+r^{2}\right)^{-1 / 2}\right]$ and have observed two kinds of solutions for Eqs. (B), (C) : If the potential well is sufficiently flat $\left(r_{0} \gtrsim \Delta s\right)$, the particle 
performs damped oscillations and comes finally to rest on the ground of the potential well $(r=0)$, when all its initial energy is radiated away. However, if the width $\left(r_{0}\right)$ of the potential well is roughly equal or less than the non-locality parameter $(\Delta s)$ entering the equation of motion, then the particle looses first energy (damped oscillations as before), but finally the motion becomes periodic and radiationless. This very interesting effect resembles in some sense the quantum theoretical, stationary states in the potential well, albeit on a different length scale.

\section{Unified Form of the Non-local Equations}

In order to compare the various solutions of Eqs. (A), (B), (C), we have to put them into a common unified form and must then specify the parameters left undetermined up to now.

As the fit parameters to be adapted to the special particle data we choose the mass ratio $\varrho$

$$
\varrho:=m_{\mathrm{el}} / m_{\text {mech }}
$$

and the non-locality parameters $\Delta s$ (resp. $\Delta \sigma$; see below). Introducing the characteristic extension parameter $\Delta s_{\exp }$ as

$$
m_{\exp } c^{2} \Delta s_{\exp }=\frac{2}{3} Z^{2}
$$

we can henceforth use the dimensionless proper time $\sigma:=s / \Delta s_{\exp }$ and obtain then from $(\mathrm{I}, 3)$ and (II, 2) for the magnitude of the non-locality expressed in the new variable $\sigma$

$$
\Delta \sigma:=\Delta s / \Delta s_{\mathrm{exp}}=m_{\mathrm{exp}} / m_{\mathrm{el}}=1+\varrho^{-1} .
$$

Furthermore, the force $K^{\lambda}$ can be put in normalized form $\left(Q^{\lambda}\right)$ by dividing it through the "surface" field strength $E^{*}=\frac{2}{3} Z^{2} / \Delta s^{2}{ }_{\text {exp }}$, which yields $Q^{\lambda}=$ $K^{\lambda} / E^{*}$. Finally, the usual ansatz for one-dimensional motion $\quad\left\{\boldsymbol{u}_{(\sigma)}^{\lambda}\right\}=\left\{\operatorname{Cosh} w_{(\sigma)} ; 0,0, \operatorname{Sinh} w_{(\sigma)}\right\} \quad$ converts the Eqs. (A), (B), (C) into their one-dimensional analogues (a), (b), (c)

$$
\begin{gathered}
w_{(\sigma-\Delta \sigma)}^{\prime} \operatorname{Cosh} \Delta_{1} w=Q_{(\sigma-\Delta \sigma)}, \\
w_{(\sigma)}^{\prime}+\varrho w_{(\sigma-\Delta \sigma)}^{\prime} \operatorname{Cosh} \Delta_{1} w=(1+\varrho) Q_{(\sigma)}, \\
w_{(\sigma)}^{\prime}+\frac{1}{2} \frac{\varrho^{2}}{1+\varrho} \operatorname{Sinh} \Delta_{2} w=(1+\varrho) Q_{(\sigma)}, \\
\left(w_{(\sigma)}^{\prime}:=\mathrm{d} w_{(\sigma)} / \mathrm{d} \sigma ;\right. \\
\left.\Delta_{1} w:=w_{(\sigma)}-w_{(\sigma-\Delta \sigma)} ; \quad \Delta_{2} w=w_{(\sigma)}-w_{(\sigma-2 \Delta \sigma)}\right) .
\end{gathered}
$$

$Q_{(\sigma)}$ is the normalized force acting along the direction of motion (here: $x^{3}$-axis).
These differential-difference equations for the auxiliary velocity $w_{(\sigma)}$ must be supplied by the proper time derivatives of the particle world line $x^{\lambda}=z_{(s)}^{\lambda}$

$$
\begin{aligned}
& \frac{\mathrm{d} z^{3}}{\mathrm{~d} s}=\operatorname{Sinh} w_{(s)}=\frac{\mathrm{d}\left(z^{3} / \Delta s_{\exp }\right)}{\mathrm{d}\left(s / \Delta s_{\exp }\right)}=: \frac{\mathrm{d} \zeta}{\mathrm{d} \sigma}=\operatorname{Sinh} w_{(\sigma)}, \\
& \frac{(\mathrm{II}, 4)}{\mathrm{d} z^{0}}=\operatorname{Cosh} w_{(s)}=\frac{\mathrm{d}\left(z^{0} / \Delta s_{\exp }\right)}{\mathrm{d}\left(s / \Delta s_{\exp }\right)}=: \frac{\mathrm{d} T}{\mathrm{~d} \sigma}=\operatorname{Cosh} w_{(\sigma)} .
\end{aligned}
$$

Together with the corresponding initial conditions, this set of equations determines the particle trajectory $\zeta=\zeta_{(T)}$.

In order to facilitate subsequent discussions, let us finally repeat here the expressions for the invariant radiation rate $\Re$, which follow easily from the three equations of motion, if they are understood to be of the form (introduce $\tau:=s / c$ )

$$
\frac{\mathrm{d} P_{\mathrm{mech}}^{\mu}}{\mathrm{d} \tau}+\frac{\mathrm{d} P_{\mathrm{el}}^{\mu}}{\mathrm{d} \tau}+\frac{\mathrm{d} P_{\mathrm{rad}}^{\mu}}{\mathrm{d} \tau}=K^{\mu}
$$

$\left[P_{\text {mech }}^{\mu} \equiv 0\right.$ for case $(\mathrm{A})$, where $\left.m_{\exp }=m_{\mathrm{el}}\right]$ and if $\Re$ is defined by

$$
\Re=u_{\mu} \mathrm{d} P_{\mathrm{rad}}^{\mu} / \mathrm{d} s .
$$

Thus, one finds for (A) and (B)

$$
\Re_{\mathrm{I}}=-m_{\mathrm{el}} c(\hat{\dot{u}} u) \Rightarrow m_{\mathrm{el}} c \dot{w}_{(s-\Delta s)} \operatorname{Sinh} \Delta_{\mathbf{1}} w_{(s)}
$$

and for (C)

$$
\begin{aligned}
& \Re_{\mathrm{II}}=-\frac{m_{\mathrm{el}} c}{2 \Delta s}(u \cdot \Delta u) \Rightarrow \frac{m_{\mathrm{el}} c}{2 \Delta s} \\
& \cdot\left\{\operatorname{Cosh} \Delta_{2} w_{(s)}-1\right\} \geqq 0 .
\end{aligned}
$$

Observe that $\Re_{\mathrm{II}}$ is positive-semidefinit in contrast to $\Re_{\mathrm{I}}$.

\section{Attractive Coulomb Force}

Let us substitute now the Coulomb force

$$
Q_{(\zeta)}=\frac{3}{2} \frac{Z^{*}}{Z} \frac{\zeta}{\mid \zeta^{3}}
$$

into Eqs. (a), (b), (c) of the foregoing section, Since Eq. (a) is of causality violating character and hence must be integrated backwards in time (as was done in Ref. ${ }^{18}$ ), we have omitted case (a) for a discussion of the motion in the attractive potential, because one does not know the final state of motion, which would work as the starting condition for the 
backward integration. Quite similar difficulties are present in the Lorentz-Dirac theory, where it is claimed that a physically reasonable solution does not exist at all ${ }^{17}$.

However, both Eqs. (b), (c) together with (III, l) have unique and well-behaved solutions, as long as the singular point $(\zeta=0)$ is excluded from the considerations. For the solution, which are plotted in Fig. 1, we have prescribed the initial condition that the particle be held fixed $(w \equiv 0)$ for $T<0$ and then be released at $T=\sigma=0$ in the starting position $\zeta_{(T=0)}=: \zeta_{0}=-5$ (mass ratio $\varrho=0.9$; charge ratio $\left.Z^{*} / Z=-1\right)$.

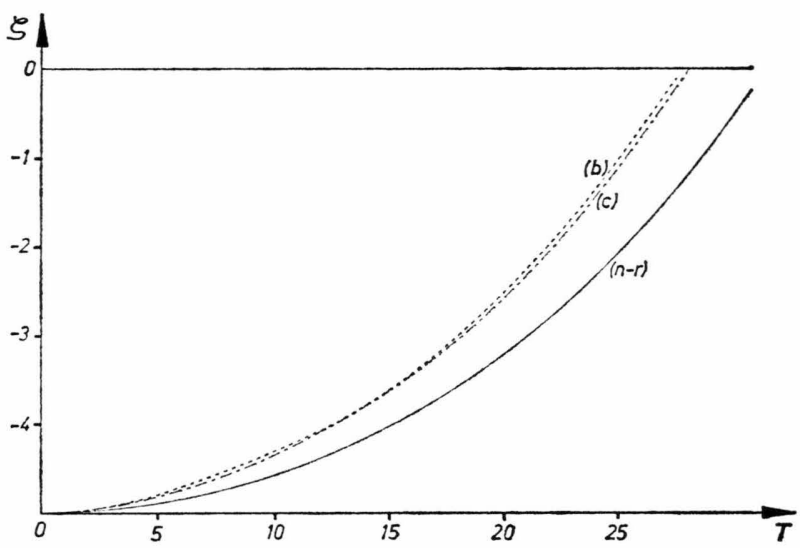

Fig. 1

As seen from Fig. 1, the result is somewhat unexpected: Despite the existence of the radiation recoil, the radiating particle due to (b) or (c) is running towards the attractive center more rapidly than a non-radiating particle described by the nonradiative equation of motion

$$
m_{\exp } c^{2} \dot{u}^{\lambda}=K^{\lambda} \Rightarrow w_{(\sigma)}^{\prime}=Q_{(\sigma)} . \quad(n-r)
$$

In order to detect the reason for this behaviour, it is only necessary to look at the local approximations for (b) and (c) [expand $w^{\prime}{ }_{(\sigma-\Delta \sigma)} \approx w_{(\sigma)}^{\prime}-\Delta \sigma w^{\prime \prime}{ }_{(\sigma)}$ in Eq. (b) and

$$
\operatorname{Sinh} \Delta_{2} w \approx \Delta_{2} w \approx 2 \Delta \sigma w_{(\sigma)}^{\prime}-\frac{1}{2}(2 \Delta \sigma)^{2} w^{\prime \prime}{ }_{(\sigma)}
$$
in Eq. (c) ] :

$$
w_{(\sigma)}^{\prime}-w_{(\sigma)}^{\prime \prime}=Q_{(\sigma)} .
$$

As stated repeatedly in the previous papers, the onedimensional (normalized) Lorentz-Dirac equation (III, 2) cannot be considered as the consistent local approximation for the original Eqs. (b), (c), because it brings in unphysical traits, which are not contained in the original equations (runaways, causality violation). Hence, we have to perform a further approximation ${ }^{14}$ in $(\mathrm{III}, 2)$ to obtain

$$
w_{(\sigma)}^{\prime}=Q_{(\sigma)}+Q_{(\sigma)}^{\prime} \text {. }
$$

But with the attractive Coulomb force (III, 1) one has (observe $\zeta<0 ; w_{(\sigma)} \geqq 0$ )

$$
Q^{\prime}{ }_{(\sigma)}=\frac{\mathrm{d} Q_{(\zeta)}}{\mathrm{d} \zeta} \cdot \operatorname{Sinh} w_{(\sigma)}=3 \frac{Z^{*}}{Z} \frac{1}{\zeta^{3}} \operatorname{Sinh} w_{(\sigma)} \geqq 0 .
$$

Thus, the additional force $Q^{\prime}{ }_{(\sigma)}$ leads to a stronger acceleration towards the attractive center.

This result is opposite to that predicted by the corresponding approximation for Eq. (a)

$$
\boldsymbol{w}_{(\sigma)}^{\prime} \approx Q_{(\sigma)}-\frac{1}{2}(\Delta \sigma)^{2} Q^{3}{ }_{(\sigma)},
$$

which is easily obtained by expanding the Coshfunction. Here the acceleration towards the center would be smaller than in the non-radiative case (n-r). We can check this result in the next section, where explicit numerical solutions of all three Eqs. (a), (b), (c) are obtained for the repulsive potential.

Finally, let us point out that one does not have to struggle here against those numerical difficulties in the Lorentz-Dirac theory, where even small numerical errors cause a transition from the single physical solution to the continuous set of runaway solutions ${ }^{17,8}$.

\section{Repulsive Coulomb Force}

For the repulsive force, we have only to change the charge ratio $Z^{*} / Z=-1$ into $Z^{*} / Z=+1$ and leave all other formulae unaltered. Releasing the particle from rest in some distance from the force center (as in the foregoing section) would be no interesting initial condition; the particle would be simply driven away from the center. Therefore, we have shot it with various initial velocities

$$
\left(w_{(\sigma)} \equiv w_{\text {in }}=\text { const. for } \sigma<0 ; \zeta \leqq \zeta_{0}\right)
$$

in some distance $\zeta_{0}$ towards the force center. Figure 2 shows a comparison between the three Eqs. (a), (b), (c) and the non-radiative solution from (n-r). The parameters chosen for this figure are $Q=0.5 ; w_{\text {in }} \approx 0.8\left(\Rightarrow v_{\text {in }} / c=\operatorname{Tanh} w_{\text {in }} \approx 0.66\right) ; \zeta_{0}$ $=-10$. 


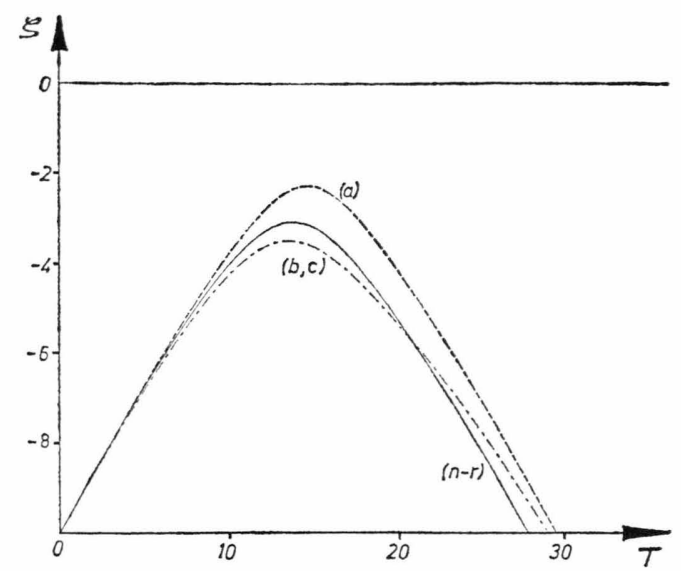

Fig. 2

From Fig. 2 one recognizes two conspicuous effects :

(1) Though the impact velocity $w_{\text {in }}$ was chosen to be the same for all four Eqs. (a), (b), (c), $(n-r)$, the solution of (a) comes closest to the force center, then follows that of (n-r). The solutions of (b) and (c), being hardly separated in this diagram, predict the most remote turning point from the repulsive center.

(2) Whereas the solutions of (b) and (c) predict a smaller absolute value of the final velocity $v_{\text {out }}$ (observe $v / c=\mathrm{d} \zeta / \mathrm{d} T$ ) than in the non-radiative case (n-r), for which $v_{\text {in }}=-v_{\text {out }}$ must be valid, Eq. (a) delivers a final velocity which equals roughly that of the non-radiative case. For impact velocities lower than that chosen for Fig. 2 the final velocity predicted by Eq. (a) is always smaller in amount than

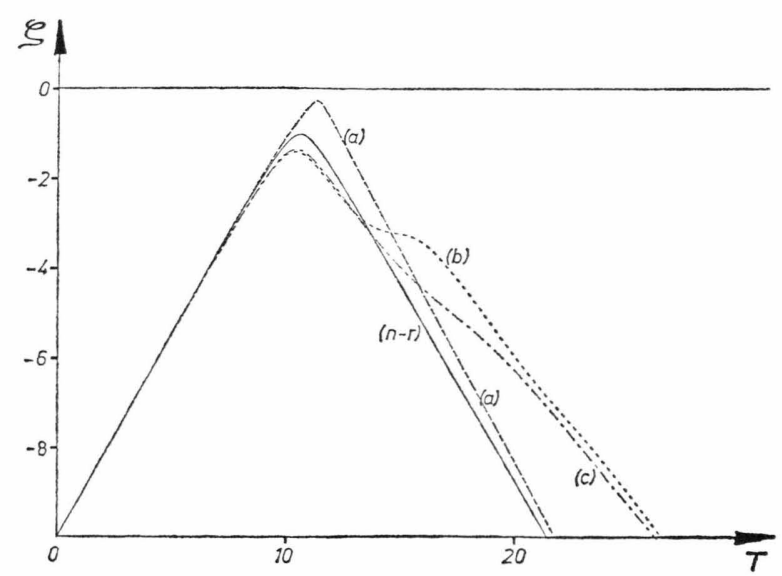

Fig. 3 that of the non-radiative case (n-r), as one would expect on account of the energy loss by radiation; whereas for higher impact velocities [see Fig. 3, where $v_{\text {in }} / c \approx 0.90$ but $\left|v_{\text {out }}\right| / c \approx 0.96$ for Eq. (a)] the particle described by (a) would retreat from the force center even more rapidly than it was originally shot in: $\left|v_{\text {out }}\right|>\left|v_{\text {in }}\right|$ !

Let us clarify these two observations now. As for point (1), we have only to compare Eq. (III, 3) as approximation for both Eq. (b) and (c) with the approximation (III, 5) for Equation (a). In the present repulsive case, one has $Q_{(\sigma)}<0$ for $\zeta<0$ and further

$$
\frac{\mathrm{d} Q_{(\sigma)}}{\mathrm{d} \sigma}=\frac{\mathrm{d} Q_{(\zeta)}}{\mathrm{d} \zeta} \operatorname{Sinh} w_{(\sigma)}>0 \text { for }\left\{\begin{array}{l}
w_{(\sigma)}>0 \\
w_{(\sigma)}<0 .
\end{array}\right.
$$

Hence, the retarding action of the repulsive center is reinforced by the radiation reaction term $Q_{(\sigma)}^{\prime}$ of Eq. (III, 3), if the particle approaches the force center; whereas the acceleration is weakened, if the particle is retreating from the center. This explains (at least for these low accelerations) why the radiating particle due to (b) and (c) keeps its distance from the center more remote as in the (n-r) case. However, it follows from the approximation (III, 5) for Eq. (a) that the radiation reaction force proportional to $Q^{3}{ }_{(\sigma)}$ is always weakening the acceleration $w_{(\sigma)}^{\prime}$ with respect to the (n-r) case, and thus the particle can come closer to the repulsive center at the end of the approach period.

In view of these considerations, the present numerical results confirm also the earlier statements ${ }^{13}$ that the Lorentz-Dirac equation (III, 2) is not the correct approximation for Eq. (a), though it can be "deduced" from it by means of expansions like (observe here $\Delta \sigma=1$ )

$$
\begin{aligned}
Q_{(\sigma)} \approx Q_{(\sigma-\Delta \sigma)}=w_{(\sigma-\Delta \sigma)}^{\prime} & \operatorname{Cosh} \Delta w \approx w^{\prime}{ }_{(\sigma-\Delta \sigma)} \\
\cdot & \approx w_{(\sigma)}^{\prime}-w^{\prime \prime}{ }_{(\sigma)} .
\end{aligned}
$$

Next, we turn to the second point (2). We shall readily show that the rather unpleasant prediction $\left|v_{\text {out }}\right|>\left|v_{\text {in }}\right|$ is not really a lack of the radiation reaction expression on the left-hand side of Eqs. (A), (a), but it stems from the ansatz for the external force ${ }^{13}$

$\left\{K_{(s)}^{\lambda}\right\}=\left(1-\frac{\boldsymbol{v}^{2}(s)}{c^{2}}\right)^{-1 / 2}\left\{\boldsymbol{K}_{(s-\Delta s)} \cdot \frac{\boldsymbol{v}_{(s)}}{c} ; \boldsymbol{K}_{(s-\Delta s)}\right\}$, $(\mathrm{IV}, 3)$ 
which we had originally preferred to the strictly local expression

$$
\left\{K_{(s)}^{\lambda}\right\}=\left(1-\frac{\boldsymbol{v}^{2}(s)}{c^{2}}\right)^{-1 / 2}\left\{\boldsymbol{K}_{(s)} \cdot \frac{\boldsymbol{v}_{(s)}}{c} ; \boldsymbol{K}_{(s)}\right\},
$$

in order to avoid pre-acceleration effects. But in view of the causality violating character of Eq. (A), it does not make things worse, if we reintroduce $(\mathrm{IV}, 4)$ and obtain instead of (a)

$$
w_{(\sigma-\Delta \sigma)}^{\prime} \operatorname{Cosh} \Delta_{1} w=Q_{(\sigma)} \text {. }
$$

It is easy to show that Eq. ( $\left.\mathrm{a}^{*}\right)$ predicts $\left|v_{\text {out }}\right|<\left|v_{\text {in }}\right|$ in the repulsive case: The energy balance of Eq. (A) with the force expression (IV, 4) instead of (IV, 3) reads for one-dimensional motion

$$
\frac{\mathrm{d} u_{(\sigma-\Delta \sigma)}^{0}}{\mathrm{~d} \sigma}-\left(u \cdot \frac{\mathrm{d} u_{(\sigma-\Delta \sigma)}}{\mathrm{d} \sigma}\right) u_{(\sigma)}^{0}=Q_{(\sigma)} \operatorname{Sinh} w_{(\sigma)} .
$$

Integration between initial (in) and final (out) states yields (observe that $Q_{(\zeta)}=-\mathrm{d} \Phi_{(\zeta)} / \mathrm{d} \zeta$ is a potential force)

$$
\begin{aligned}
u_{\text {out }}^{0}-u_{\text {in }}^{0_{\text {in }}}- & \int_{\text {in }}^{\text {out }}\left(u_{(\sigma)} \cdot \frac{\mathrm{d} u_{(\sigma-\Delta \sigma)}}{\mathrm{d} \sigma}\right) \cdot \mathrm{d} T \\
& =-\int_{\text {in }}^{\text {out }} \mathrm{d} \sigma \frac{\mathrm{d} \zeta_{(\sigma)}}{\mathrm{d} \sigma}\left(\frac{\mathrm{d} \Phi_{(\zeta)}}{\mathrm{d} \zeta}\right)_{(\sigma)},
\end{aligned}
$$

where the right-hand side vanishes for the asymptotic states $\left(\Phi_{\text {out }}=\Phi_{\text {in }}\right)$

$$
u_{\text {out }}^{0}-u_{\text {in }}^{\mathbf{o}_{\text {in }}}=\int_{\text {in }}^{\text {out }}\left(\boldsymbol{u}_{(\sigma)} \cdot \frac{\mathrm{d} \boldsymbol{u}_{(\sigma-\Delta \sigma)}}{\mathrm{d} \sigma}\right) \mathrm{d} T,(\mathrm{IV}, 7)
$$

or specialized for one-dimensional motion

$$
u_{\text {out }}^{0}-u_{\text {in }}^{0}=-\int_{\text {in }}^{\text {out }} Q_{(\sigma)} \operatorname{Tanh} \Delta_{1} w_{(\sigma)} \mathrm{d} T .
$$

Since in the repulsive case $\left(Q_{(\sigma)}<0\right)$ the velocity is always decreasing $(\Delta w<0)$ for $\zeta<0$, we have

$$
u_{\text {out }}^{0}<u_{\text {in }}^{0} \Rightarrow\left|v_{\text {out }}\right|<\left|v_{\text {in }}\right| .
$$

This satisfactory result refers to Equation $\left(a^{*}\right)$. Indeed, even for very high impact velocities the results of Eq. ( $\left.\mathrm{a}^{*}\right)$ never violate (IV, 9) [see Fig. 4, where $v_{\text {in }} / c \approx 0.9935$ and $\left|v_{\text {out }}\right| / c \approx 0.8000$ for Equation $\left.\left(\mathrm{a}^{*}\right)\right]$.

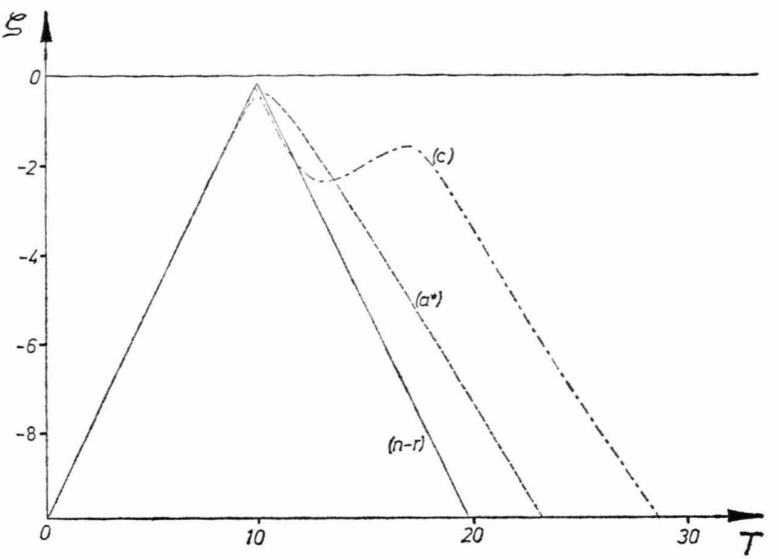

Fig. 4

Quite similar manipulations lead to the analogous "conservation laws" for Eq. (b)

$$
u_{\text {out }}^{0}-u_{\text {in }}^{0}=-\frac{\varrho}{1+\varrho} \int_{\text {in }}^{\text {out }} w_{(\sigma-\Delta \sigma)}^{\prime} \operatorname{Sinh} \Delta_{1} w_{(\sigma)} \mathrm{d} T
$$

and for Eq. (c)

$$
\begin{aligned}
u_{\text {out }}^{0}-u_{\text {in }}^{0}= & -\frac{1}{2} \frac{\varrho^{2}}{(1+\varrho)^{2}} \\
& \cdot \int_{\text {in }}^{\text {out }}\left(\operatorname{Cosh} \Delta_{2} w_{(\sigma)}-1\right) \mathrm{d} T \leqq 0 .
\end{aligned}
$$

From here we recognize readily that only Eq. $(\mathrm{IV}, 8 \mathrm{c})$ leads strictly to $(\mathrm{IV}, 9)$; whereas Eq. $(\mathrm{IV}, 8 \mathrm{~b})$ violates $(\mathrm{IV}, 9)$ for sufficiently high impact velocities (see Fig. 5 , where $v_{\text {in }} / c \approx 0.95$; $\left|v_{\text {out }}\right| / c \approx 0.98$ ).

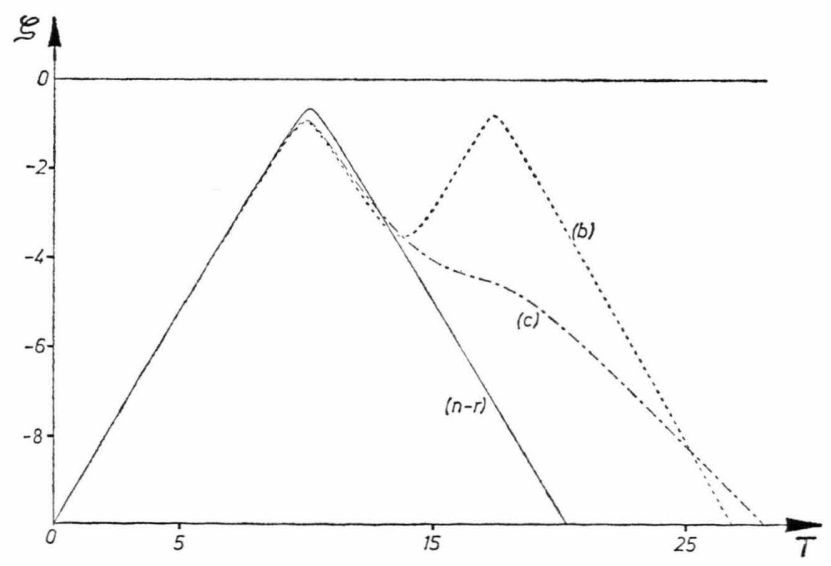

Fig. 5 
So we see that the Equation of motion (C) is a very agreeable one with respect to the validity of $(\mathrm{IV}, 9)$, because the invariant radiation rate (II, 9) is always positive or zero.

Finally, let us point out an effect, which is clearly visible in Figs. 4 and 5: The particle is first rejected by the repulsive center and then approaching once more, until it is thrown outwards definitively. As a consequence, the particle remains in the neighbourhood of the force center for a relatively long time. In some sense, this behaviour resembles a sort of intermediate "compound state". This effect arises however only for sufficiently high impact velocities.

\section{Modified Coulomb Potential}

As indicated earlier ${ }^{15}$, the equations of motion (A), (B), (C) admit stationary radiationless motions characterized by $\Re_{I}=\Re_{I I}=0$. In order to study such motions, we first have to remove the Coulomb singularity from the one-dimensional trajectory of the particle (this would not be necessary for two-dimensional motion), because the motion should not pass through a singular point.

Hence, we substitute the non-singular force

$$
Q_{(\zeta)}=\frac{3}{2} \frac{Z^{*}}{Z} \zeta\left(\zeta_{1}^{2}+\zeta^{2}\right)^{-3 / 2} ; \quad Z^{*}=-Z
$$

for $Q_{(\sigma)}$ into Eqs. (b), (c) [case (a) is omitted here for the same reasons as in Section III]. The same initial conditions as in Sect. III are used also here.

First, we have studied a relatively flat potential well for both Eqs. (b), (c) in Fig. $6\left(\zeta_{1}{ }^{2}=12\right.$; $\left.\varrho=0.7 \mid \Rightarrow \zeta_{1} \approx 1.4 \Delta \sigma\right)$. The particle was released from rest at $\zeta_{0}=-7\left(\approx-2\left|\zeta_{1}\right|\right)$. It happens ex-

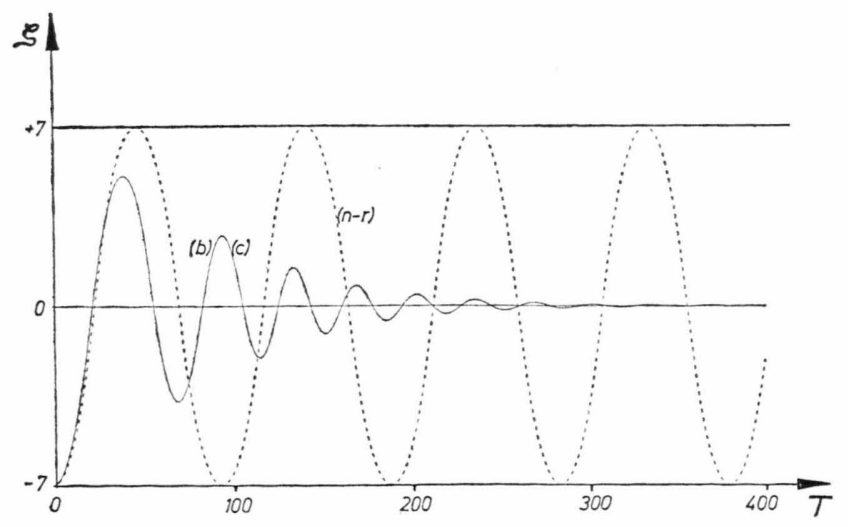

Fig. 6 actly what one would except in this case: The particle performs damped oscillations in the potential well until it comes to rest on the ground of the well, when all its initial energy is radiated away.

But now choosing a narrow well (for Fig. 7: $\left.\zeta_{0}=-3.4 ; \zeta_{1}=1 ; \varrho=0.1 \mid \Rightarrow \zeta_{1}=\frac{1}{11} \Delta \sigma\right)$, one recognize in Fig. 7, that the particle motion is stabilized at constant amplitude after the latter has decreased for a few initial oscillations. The stationary oscillation recovered in Fig. 7 is however not the only one in this potential well. We have rised the initial separation $\zeta_{0}$ from $\zeta_{0}=-3.4$ of Fig. 7 to $\zeta_{0}=-3.8$ for Fig. 8 (all other parameters unchanged) and then a stationary state is found with larger amplitude. The ratio of the two stationary amplitudes of Fig. 7 and Fig. 8 is roughly $1: 1.75$. Next we have chosen $\zeta_{0}$ to be situated between the corresponding values for Fig. 7 and Fig. 8, namely $\zeta_{0}=3.6$, and we see from the resulting Fig. 9 that the particle is now oscillating for a longer time in the higher state of Fig. 8 until it falls down to the

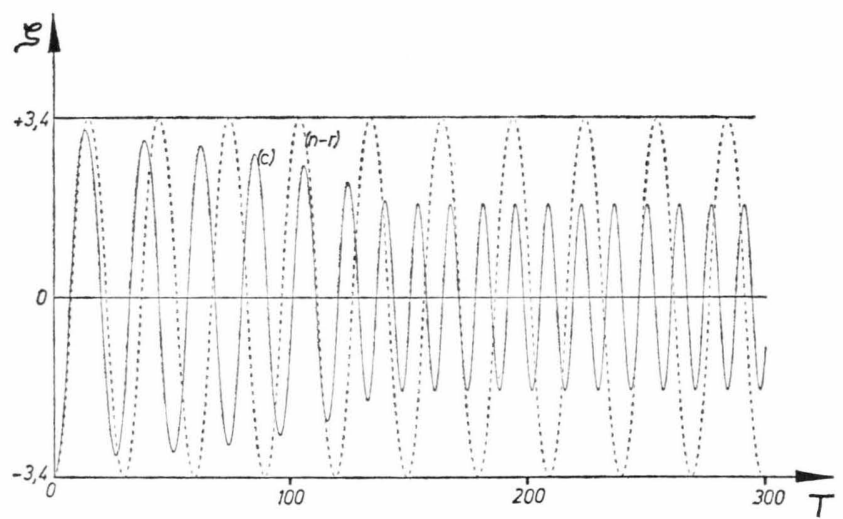

Fig. 7

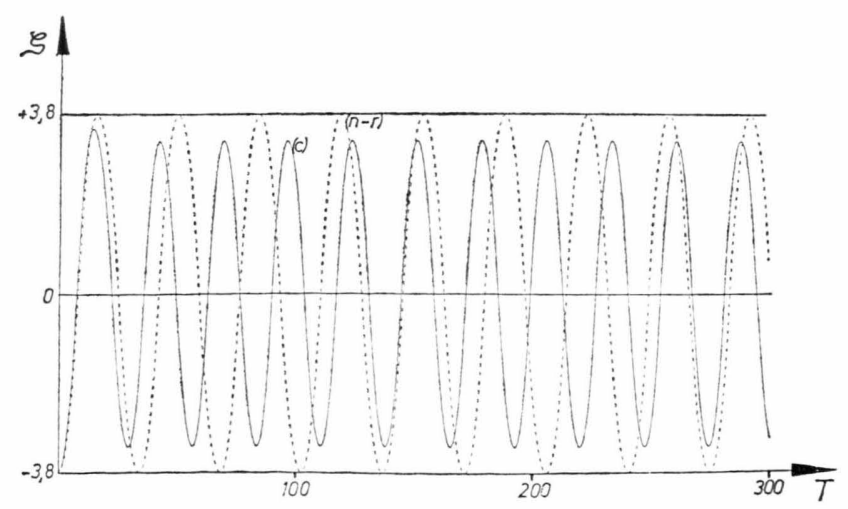

Fig. 8 


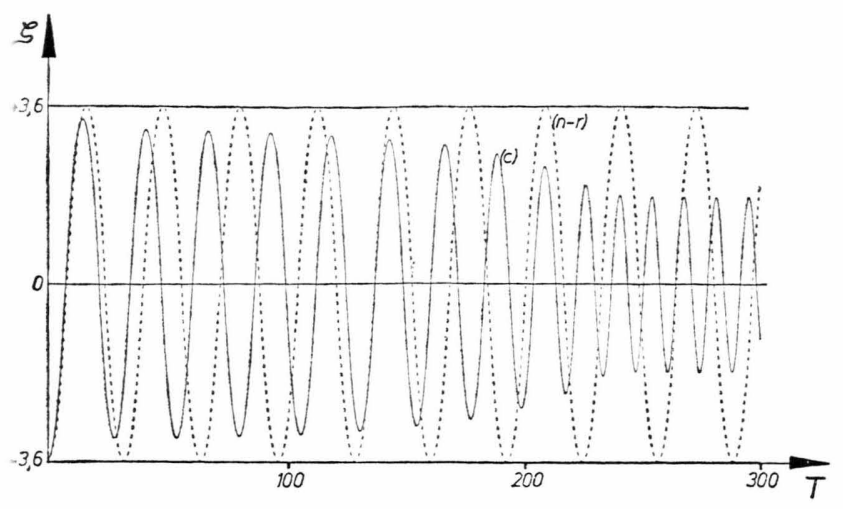

Fig. 9

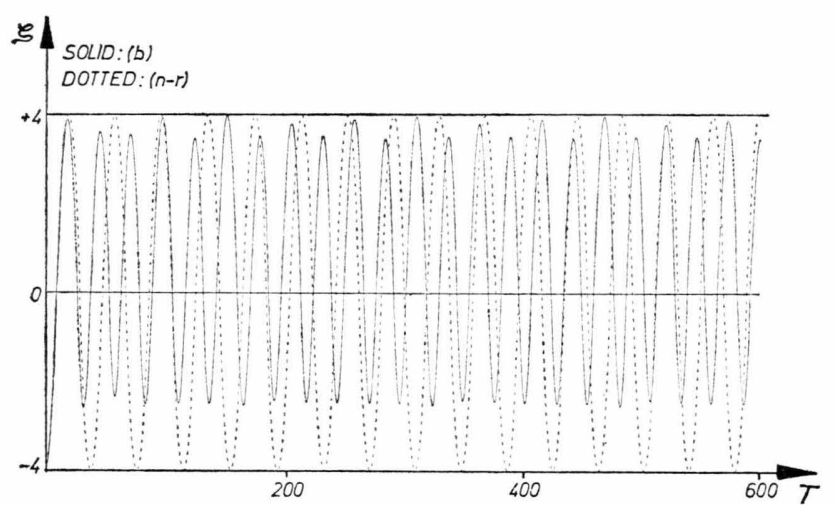

Fig. 10

1 P. Caldirola, Nuovo Cim. 3, Suppl. 2, 297 [1956].

2 D. Gromes and J. Petzold, Z. Phys. 198, 79 [1967]; ibid. 199, 299 [1967].

3 W. L. Burke, Phys. Rev. A 2, 1501 [1970].

4 T. C. Mo and C. H. Papas, Phys. Rev. D 4, 3566 [1971].

5 W. B. Bonnor, Proc. Roy. Soc. London A 337, 591 [1974].

6 R. L. Ingraham, Nuovo Cim. 27 B, 293 [1975].

7 W. E. Baylis and J. Huschilt, Phys. Rev. D 13, 3237 [1976].

8 J. C. Kasher, Phys. Rev. D 14, 939 [1976].

9 H. Stöckel, Fortschr. Phys. 24, 417 [1976].

10 J. C. Herrera, Phys. Rev. D 15, 453 [1977].

11 P. A. M. Dirac, Proc. Roy. Soc. London A 167, 148 [1938]. lower state of Figure 7. It seems that one can hold the particle arbitrarily long on the upper state by choosing appropriate initial conditions.

The stationary states discussed so far refer to Eq. (c), and we want to study now the analogous effects for Equation (b). Figure 10 shows the results for the parameter choice $\zeta_{0}=-4 ; \zeta_{1}{ }^{2}=2 ; \varrho=0.1$. As a striking feature in this figure, one recognizes a certain irregularity in comparison with the corresponding Figs. 7-9 for Equation (c). This irregularity might be due to the fact that the radiation rate $\Re_{\mathrm{II}}$ of formula (II, 8) is not positive-definit. Therefore, a stationary state must not necessarily be described by the non-radiative Eq. (n-r) with $\Re_{\text {II }} \equiv 0$, but it can have $\Re_{\text {II }} \neq 0$ such that only the average of the radiated energy over several oscillations becomes zero. It is then possible that the radiated energy emitted during one half-period is reabsorbed during the following half-oscillation, and thus the amplitude of the oscillations exhibits itself oscillatory character.

An even more peculiar consequence is that the irregular oscillations are not "centered" around the potential minimum $(\zeta=0)$ but seem to be shifted upwards in the diagram. In view of the limited computer time, we have not succeeded in finding the initial conditions, which would lead to regular oscillations $\left(\Re_{\mathrm{II}} \equiv 0\right)$ as they occur in the non-radiative case (n-r) and in Eq. (c), though the above considerations show that such oscillations must be possible also for Equation (b).

12 F. Rohrlich, Classical Charged Particles, Addison-Wesley, Reading, Mass. 1965.

13 M. Sorg, Z. Naturforsch. 31 a, 664 [1976]; ibid. 31 a, 1133 [1976].

14 M. Sorg, Z. Naturforsch. 32 a, 101 [1977]; ibid. 32 a, 383 [1977].

15 M. Sorg, Z. Naturforsch. 32 a, 659 [1977].

16 J. Petzold and M. Sorg, Generalized Caldirola Equations for the Classically Radiating Electron, submitted for publication in Z. Physik.

17 W. E. Baylis and J. Huschilt, Phys. Rev. D 13, 3262 [1976].

18 P. Alber, W. Heudorfer, and M. Sorg, Z. Naturforsch. 32 a, 319 [1977]. 